\title{
Association between extranodal natural killer/T-cell lymphoma and hepatitis B viral infection: a case-control study
}

Kefeng Wang 1,2,3,4*, Hang Yang 1,2,3*, Wenjun He ${ }^{5 *}$, Yi Xia 1,2,3, Zhongjun Xia 2,3,6, Su Li 2,3,7, Huiqiang Huang 1,2,3, Zhiming Li 1,2,3, Panpan Liu 1,2,3凶, and Wenqi Jiang 1,2,3凶

1. Department of Medical Oncology. Sun Yat-sen University Cancer Center.

2. State Key Laboratory of Oncology in South China.

3. Collaborative Innovation Center for Cancer Medicine. Guangzhou, P.R. China.

4. Department of Thoracic Surgery, The Sun Yat-sen Memorial Hospital of Sun Yat-sen University. Guangzhou, P.R. China.

5. Department of Medical Statistic and Epidemiology, School of Public Health, Sun Yat-sen University.

6. Department of Hematological Oncology. Sun Yat-sen University Cancer Center.

7. Clinical Trial Center. Sun Yat-sen University Cancer Center.

${ }^{*}$ Kefeng Wang, Hang Yang and Wenjun He contributed equally to this article.

$\triangle$ Corresponding authors: Wenqi Jiang, Department of Medical Oncology. State Key Laboratory of Oncology in South China. Sun Yat-sen University Cancer Center, Collaborative Innovation Center for Cancer Medicine, 510060 Guangzhou, Guangdong, P.R. China. Tel: 86-20-87343349; Fax: 86-20-87363349; E-mail: jwqsysucc@163.com Or Panpan Liu, Department of Medical Oncology. State Key Laboratory of Oncology in South China. Sun Yat-sen University Cancer Center, Collaborative Innovation Center for Cancer Medicine, 510060 Guangzhou, Guangdong, P.R. China. Tel:86-20-87343571; Fax: 86-20-87343571;

E-mail:liupp@sysucc.org.cn

(C) Ivyspring International Publisher. This is an open access article distributed under the terms of the Creative Commons Attribution (CC BY-NC) license (https://creativecommons.org/licenses/by-nc/4.0/). See http://ivyspring.com/terms for full terms and conditions.

Received: 2017.02.14; Accepted: 2017.05.19; Published: 2017.08.22

\begin{abstract}
Extranodal natural killer/T-cell lymphoma, nasal type (ENKTL) is a rare subtype of lymphoma that is often associated with poor clinical prognosis. Several studies have shown that hepatitis B virus (HBV) infection may be associated with increased risk of B-cell non-Hodgkin lymphoma; however, because of the rarity of ENKTL, little is known about its association with HBV. Our study aimed to assess whether HBV infection was associated with increased odds of ENKTL. We conducted a hospital-based case-control study including 417 ENKTL cases and 488 age- and sex-matched subjects with nonmalignant diseases unrelated to HBV infection. Multivariable unconditional logistic regression analyses were performed to estimate adjusted odds ratios [AOR] and their corresponding $95 \%$ confidence intervals $(\mathrm{Cl})$. The results of the multivariable analysis showed that after adjustment for a set of known risk factors, patients previously infected with HBV (HBsAg-seronegative/ anti-HBc-seropositive) and naturally immune to HBV (anti-HBs-seropositive/anti-HBc-seropositive) were at significantly greater odds of being diagnosed with ENKTL (AOR, 1.497; 95\% Cl 1.098-2.042, $P=0.033$ and $A O R, 1.871 ; 95 \% \mathrm{Cl} 1.302-2.689, P=0.001$, respectively). After adjusting for other factors, significantly greater odds of being diagnosed with ENKTL were observed among cases who reported ever drinking alcohol (AOR, 1.675; 95\% Cl 1.054-2.660, $P=0.029)$. The odds of ENKTL diagnosis were not significantly associated with $\mathrm{ABO}$ blood type, cigarette smoking status or family history of cancer. The results of our study suggest that patients previously infected with HBV and naturally immune to $\mathrm{HBV}$ were at greater odds of being diagnosed with ENKTL.
\end{abstract}

Key words: case control study, hepatitis B viral, immune response, previously infect, natural killer/T-cell lymphoma

\section{Introduction}

Extranodal natural killer/T-cell lymphoma, nasal type (ENKTL) is a unique subtype of lymphoma that is often associated with poor clinical prognosis.[1]
ENKTL is relatively prevalent in East Asia, Mexico and South America, accounting for between 5\% and $15 \%$ of all lymphomas identified in these countries, $[2$, 
3] but quite rare in Caucasian populations, accounting for only $0.5 \%$ of the non-Hodgkin lymphomas (NHL) identified in the United States.[4,5] The results of population-based studies have supported the notion that both the genetic and environmental risk factors play a role in the incidence of ENKTL.[6] By performing comparative genomic hybridization, researchers have discovered that deletions on chromosome 6q21 may lead to downregulation of tumor suppressor genes in ENKTL malignancies.[7, 8] Somatic mutations of JAK3,[9] TP53,[10, 11] and $D D X 3 X$ [12] were detected in NKTL via genome sequencing in previous studies, thereby implicating several specific pathways (JAK-STAT, NF-kB, and MAPK) in tumor pathogenesis. Besides, Hiroyuki et al. reported HLA-class I phenotype is associated with the EBV-lymphomagenesis, because HLA-A alleles but HLA-A*0201 is in relation with escape of immune surveillance by host cytotoxic lymphocyte to latent membrane proteins (LMPs) of EBV.[13] Some environmental factors have been reported played causative role in ENKTL. Previous epidemiological studies showed an increase in the risk of developing ENKTL among individuals exposed to pesticides.[14, 15] ENKTL has been found to have a strong association with Epstein-Barr virus (EBV), and the EBV-encoded early small RNAs (EBERs) have been concurrently detected in ENKTL tumor cells.[16, 17] Additionally, recent studies have indicated that the presence of a high EBV-DNA load in plasma was positively associated with clinical stage and negatively associated with survival.[18, 19] However, discussions regarding the roles of other viral infections in the progression of ENKTL have been limited.

Hepatitis B virus (HBV) is a hepatotropic virus that can also infect and replicate in lymphoid cells.[20] $\mathrm{HBV}$ infection is highly prevalent in the Asia-Pacific region, especially in China.[21] According to recently published data, HBsAg-positive subjects are account for $7.2 \%$ in the general population.[22] A large number of studies have suggested that chronic HBV infection may play a role in the etiology of tumors, such as hepatocellular carcinoma, cholangiocarcinoma and B-cell non-Hodgkin lymphoma (NHL).[23-27] An anti-HBs immune response can be induced by vaccination [anti-HBs-(+)/anti-HBc-(-)] or by a natural response to $\mathrm{HBV}$ exposure [anti-HBs-(+)/anti-HBc-(+)]. One study found anti-HBs seropositivity to be inversely associated with B-cell NHL.[25] Some previous studies showed that ENKTL patients with HBsAg-positive status was associated with inferior OS and PFS [28] and might increase the risk of acute pancreatitis during the treatment[29], however, no case-control study had addressed the association between ENKTL and HBV infection or HBV-induced immune responses, as the performance of such studies has been limited by the low incidence of ENKTL. Furthermore, Ye and colleagues recently published a case-control study in which a significant association between HBV infection and nasopharyngeal carcinoma was identified, which, similar to ENKTL, is an EBV-related malignancy.[30] This finding indicated that interactions between HBV and EBV may affect tumorigenesis.

To this end, we hypothesized that HBV infection might be associated with increased odds of ENKTL in southern China. We conducted a hospital-based case-control study to test this hypothesis.

\section{Materials and Methods}

\section{Study population}

The present study was a longitudinal cohort study of 417 patients with newly diagnosed ENKTL at the Sun Yat-sen University Cancer Center in Guangdong, China from 2004 January to 2015 December. All patients included in the study had a diagnosis of ENKTL that has been histologically confirmed according to the 2008 World Health Organization (WHO) criteria. All the patients meet the typical features included positive in the detection of CD3, CD56, and cytotoxic molecules (such as TIA-1, perforin, and granzyme B) by immunohistochemical, and EBERs by in situ hybridization in NK/T cell. The control group consisted of patients who had sought care for nonmalignant diseases at the same hospital. Patients with nonmalignant diseases were identified based on the assignment of discharge diagnoses believed to be irrelevant to HBV infection, including breast fibroadenomas, benign adrenal tumors, gallbladder polyps, hepatic cysts, renal cysts, polycystic kidney disease, benign neoplasms of the thyroid gland, and uterine fibroids. A total of 488 ageand sex- matched eligible benign tumor controls were selected at random. All cases in ENKTL and control groups were free of infection of human immunodeficiency virus (HIV), had no immunodeficiency diseases or usage of any immunosuppressive agents, and never received blood transfusion.

This case-control study was conducted in accordance with the Helsinki Declaration and the guidelines of the Institutional Review Board of our center.

\section{Data collection}

Clinical data for all ENKTL patients and control cases were gathered from medical records, including sex, age, smoking status (ever or never), ABO blood 
type, alcohol drinking (ever or never), family history of other cancer and year of diagnosis.

\section{Laboratory test for HBV infection}

A 3-mL blood sample was routinely collected from both case and control subjects prior to the provision of any treatment, and samples were submitted to the clinical laboratories of our hospital to test for HAV, HBV, HCV, HDV, HEV, and HIV infection. Plasmas levels of hepatitis B surface antigen (HBsAg), antibodies to HBsAg (anti-HBs), hepatitis B e antigen (HBeAg), antibodies to HBeAg (anti-HBe), and hepatitis B core antibody (anti-HBc) were measured using an ELISA kit (Kehua Bio-Engineering, Shanghai, China). Assay quality control (QC) was performed according to the protocols provided by the manufacturer. In addition, we performed daily routine external quality assessments of HBV serology testing using pooled serum provided by the Ministry of Health of the PR China.

The results of the aforementioned HBV assays were used to classify patients into the following groups: HBsAg seronegative and anti-HBc seronegative patients were classified as never infected with HBV; HBsAg seropositive and anti-HBC seropositive patients were classified as chronic carriers; and anti-HBc seropositive but HBsAg seronegative patients were classified as previously infected with HBV (resolved hepatitis B).[30, 31]

Patients were categorized into HBV immune response groups using the anti-HBs and anti-HBc results. Patients were considered to be naturally immune to HBV if they were both anti-HBs and anti-HBc seropositive. Patients were considered to lack an immune response to HBV when they were both anti-HBs seronegative and anti-HBc seropositive. Patients who were anti-HBs seropositive and anti-HBc seronegative were considered to have become HBV immune following vaccination.[32]

\section{Statistical analysis}

All statistical analyses were performed using SPSS statistical package version 24.0 (SPSS Inc., Chicago, IL). Statistical significance was defined as a $P$-value less than 0.05 by two-tailed test. Pearson's $\chi^{2}$ tests and Student's t-tests were used to compare the baseline parameters between cases and controls. Unconditional logistic regression analysis was used to evaluate the associations between $\mathrm{ABO}$ blood type, HBV infection status, smoking status (ever or never), alcohol drinking status (ever or never), and family history of other cancers with ENKTL status. Multivariable unconditional logistic regression analyses were performed to assess the odds ratios
[ORs], adjusted odds ratios [AORs] and their corresponding 95\% confidence intervals [CIs] for HBV infection status after adjustment for age, sex, ABO blood type, cigarette and alcohol consumption and family history of cancer.

\section{Results}

\section{Characteristics of ENKTL patients and cancer-free controls}

A total of 417 ENKTL patients and 488 cancer-free controls were included in this case-control study. The demographic characteristics of included subjects, including gender and age, are described in Table 1 . Of the 417 ENKTL patients, $67.4 \%$ were male and $32.6 \%$ were female. As expected, the sex distribution of controls was comparable to that of ENKTL patients $(P=0.481)$. The mean age $( \pm S D)$ of the ENKTL patients was $42.25( \pm 14.68)$ years for ENKTL cases, and the mean age $( \pm \mathrm{SD})$ of the controls was $42.78( \pm 14.76)$ years. The age distributions of the ENKTL patients and controls did not differ significantly $(P=0.756)$.

Table 1. Baseline characteristics of study populations

\begin{tabular}{|c|c|c|c|c|c|}
\hline \multirow[t]{2}{*}{ Variable } & \multicolumn{2}{|c|}{ Cases $(\mathrm{N}=417)$} & \multicolumn{2}{|c|}{ Controls $(\mathrm{N}=488)$} & \multirow[t]{2}{*}{$p$} \\
\hline & No. & $\%$ & No. & $\%$ & \\
\hline Sex & & & & & 0.481 \\
\hline Male & 281 & 67.4 & 318 & 65.2 & \\
\hline Female & 136 & 32.6 & 170 & 34.8 & \\
\hline Age, y & & & & & 0.756 \\
\hline$<18$ & 14 & 3.4 & 19 & 3.9 & \\
\hline $18-39$ & 169 & 40.5 & 182 & 37.3 & \\
\hline $40-59$ & 176 & 42.2 & 212 & 43.4 & \\
\hline$>60$ & 58 & 13.9 & 75 & 15.4 & \\
\hline
\end{tabular}

\section{HBV infection status and the odds of ENKTL diagnosis}

Detailed HBV serology data are included in Table 2. In the unadjusted model, $\mathrm{HBcAb}$ seropositive patients were at 1.34 times greater odds of being diagnosed with ENKTL than their $\mathrm{HBcAb}$ seronegative counterparts $(95 \%$ CI 1.041-1.786, $P=0.024)$. In the multivariable model, anti-HBc- $(+)$ patients were also more likely to be diagnosed with ENKTL (AOR, 1.352; 95\% CI 1.025-1.782, $P=0.033$ ) than anti-HBc-(-) patients after adjusting for other factors. However, the odds of ENKTL diagnosis were not associated with $\mathrm{HBsAg}$, anti-HBs, HBeAg and anti-HBe status.

The results of the unconditional logistic regression analysis revealed that patients who had been previously exposed to HBV were at significantly greater odds of being diagnosed with ENKTL (AOR, 1.497; 95\% CI 1.098-2.042, $P=0.033$ ). Conversely, no association between chronic HBV carrier status and 
the odds of ENKTL diagnosis was identified (AOR, 1.092; 95\% CI 0.708-1.683, $P=0.690$ ).

We then compared the correlation of HBV infection status and clinical characteristics of patients with ENKTL. As shown in table 3, HBV infection status was found to be in significant correlation with Ann Arbor stage $(P=0.044)$, ALT elevation $(P=$ $0.024)$, AST elevation $(P=0.004)$.

\section{Immune response to $\mathrm{HBV}$ and the odds of ENKTL diagnosis}

In the model comparing ENKTL patients with cancer-free controls, we found that individuals who were naturally immune to $\mathrm{HBV}$ were significantly more likely diagnosed ENKTL (AOR, 1.871; 95\% CI 1.302-2.689, $P=0.001$ ). However, a lack of immune response to $\mathrm{HBV}$ was not associated with increased ENKTL odds (AOR, 1.193; 95\% CI 0.803-1.773, $P=0.383$ ). In the analysis of the effect of vaccination-associated immunity, we found that individuals who were not been immunized by vaccination were more likely to be diagnosed with ENKTL (AOR, 1.503; 95\% CI 1.139-1.985, P=0.004).

Table 2. The associations of HBV infection with the risk of NK/T cell lymphoma.

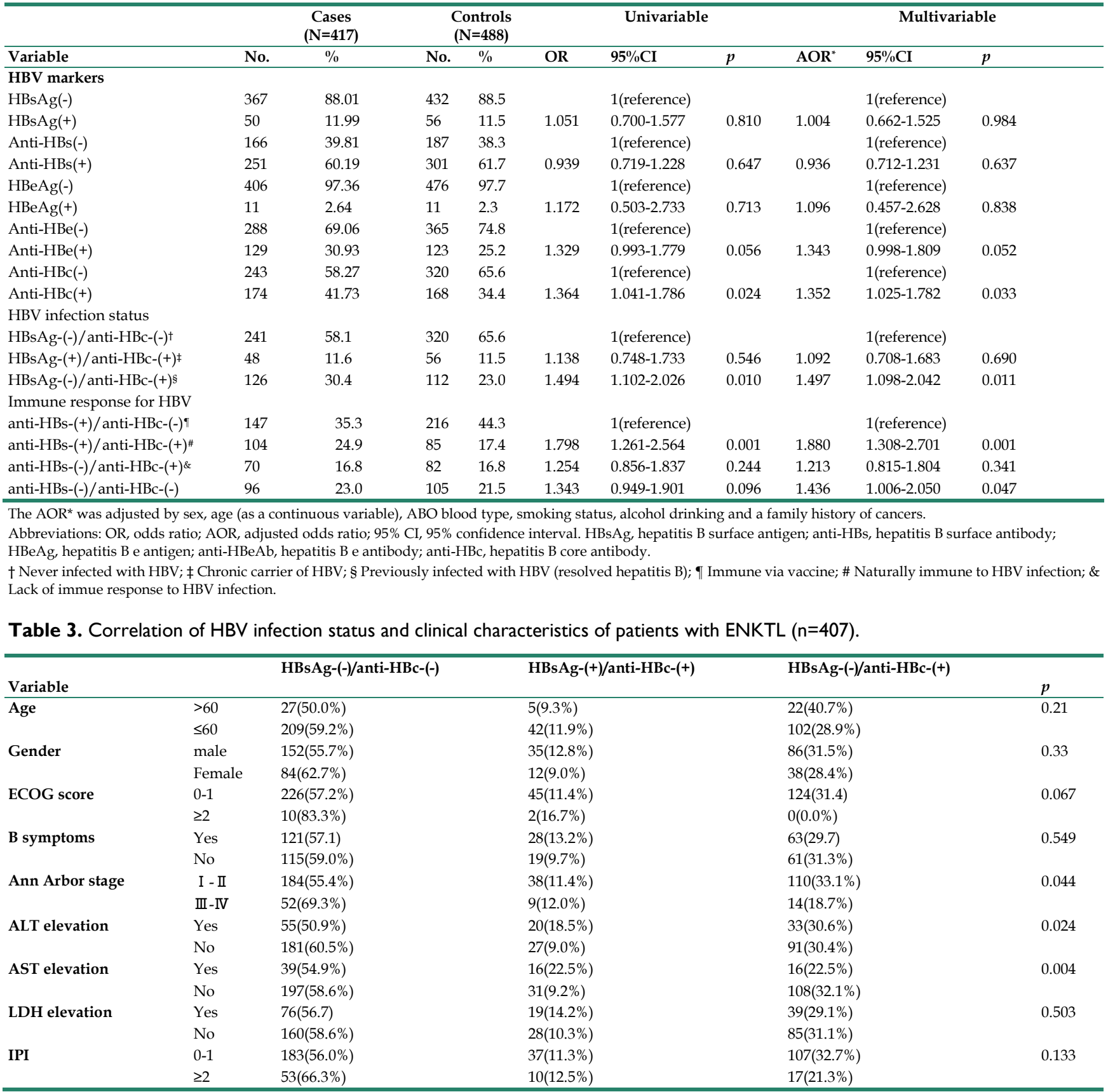




\section{Environmental and hereditary factors and the odds of ENKTL diagnosis}

We then analyzed the associations between environmental and hereditary factors and the odds of ENKTL diagnosis (Table 4). After adjusting for other factors, cases who reported ever drinking alcohol were at 1.675 times greater odds of ENKTL diagnosis than were cancer-free controls (95\% CI 1.054-2.660, $P=0.029$ ). However, $\mathrm{ABO}$ blood type, cigarette smoking and first-degree relatives of other cancers were not significantly associated with ENKTL.

In the multivariable model including age, gender, $\mathrm{ABO}$ blood type, $\mathrm{HBV}$ infection status, $\mathrm{HBV}$ immune response, cigarette smoking, alcohol drinking and family history of other cancers, the variable for the interaction between anti-HBc serostatus and alcohol drinking status was found to be significantly associated with the odds of ENKTL diagnosis (Table 5). In the adjusted model, anti-HBc-(-) subjects who reported ever drinking and anti-HBc- $(+)$ subjects were at $2.013 \quad(95 \%$ CI 1.124-3.604, $P=0.019)$ and 1.598 (95\% CI 1.149-2.222, $P=0.005)$ times greater odds of ENKTL diagnosis, respectively, when compared with anti-HBc-(-) subjects who reported never drinking. The greatest AOR was observed among subjects who were both anti-HBc-(+) and reported ever drinking (2.123, 95\% CI 1.121-4.020, $P=0.021)$.

\section{Discussion}

To our knowledge, this is the largest case-control study to evaluate the association HBV infection and the odds of ENKTL diagnosis to date. The results of this study demonstrated that patients who had been previously infected with HBV or were naturally immune to HBV were at substantially increased odds of ENKTL diagnosis. Furthermore, the association between HBV and ENKTL status was independent of a number of potential confounders, including age, gender, $\mathrm{ABO}$ blood type, cigarette smoking, alcohol consumption and family history of cancer. In addition, in this study, we confirmed previous findings suggesting that alcohol drinking was associated with the odds of ENKTL diagnosis. However, associations between ABO blood type, smoking status and family history of cancer and ENKTL status have not been well-established.

Table 4. The associations of $A B O$ blood type, cigarette smoking, alcohol drinking and family history of cancer with the risk of NK/T cell lymphoma.

\begin{tabular}{|c|c|c|c|c|c|c|c|c|c|c|}
\hline \multirow[b]{2}{*}{ Variable } & \multicolumn{2}{|c|}{$\begin{array}{c}\text { Cases } \\
(\mathrm{N}=417)\end{array}$} & \multicolumn{2}{|c|}{$\begin{array}{c}\text { Controls } \\
(\mathrm{N}=488)\end{array}$} & \multicolumn{3}{|c|}{ Univariable } & \multicolumn{3}{|c|}{ Multivariable } \\
\hline & No. & $\%$ & No. & $\%$ & OR & $95 \% \mathrm{CI}$ & $p$ & AOR $^{*}$ & $95 \% \mathrm{CI}$ & $p$ \\
\hline \multicolumn{11}{|c|}{ ABO blood type } \\
\hline A & 98 & 24.69 & 132 & 27.0 & & 1(reference) & & & 1(reference) & \\
\hline B & 101 & 25.44 & 122 & 25.0 & 1.115 & $0.769-1.616$ & 0.565 & 1.096 & $0.752-1.597$ & 0.635 \\
\hline $\mathrm{AB}$ & 32 & 8.06 & 31 & 6.4 & 1.390 & $0.795-2.431$ & 0.248 & 1.387 & $0.788-2.441$ & 0.257 \\
\hline $\mathrm{O}$ & 166 & 41.81 & 203 & 41.6 & 1.101 & $0.790-1.535$ & 0.569 & 1.081 & $0.772-1.513$ & 0.650 \\
\hline \multicolumn{11}{|c|}{ Cigarette smoking } \\
\hline Never & 316 & 75.8 & 367 & 75.2 & & 1(reference) & & & 1(reference) & \\
\hline Ever & 101 & 24.2 & 121 & 24.8 & 0.969 & $0.715-1.314$ & 0.841 & 0.762 & $0.517-1.123$ & 0.762 \\
\hline \multicolumn{11}{|c|}{ Alcohol drinking } \\
\hline Never & 352 & 84.4 & 433 & 88.7 & & 1(reference) & & & 1(reference) & \\
\hline Ever & 65 & 15.6 & 55 & 11.3 & 1.454 & $0.989-2.138$ & 0.057 & 1.675 & $1.054-2.660$ & 0.029 \\
\hline \multicolumn{11}{|c|}{ Family history of cancer } \\
\hline No & 383 & 91.8 & 453 & 92.8 & & 1(reference) & & & 1(reference) & \\
\hline Yes & 34 & 8.2 & 35 & 7.2 & 1.149 & $0.703-1.878$ & 0.579 & 1.194 & $0.719-1.983$ & 0.494 \\
\hline
\end{tabular}

The $\mathrm{AOR}^{*}$ was adjusted by sex, age (as a continuous variable), ABO blood type, HBV infection status, smoking status, alcohol drinking and a family history of cancers. Abbreviations: OR, odds ratio; AOR, adjusted odds ratio; 95\% CI, 95\% confidence interval.

Table 5. The associations of HBV infection status and alcohol drinking with the risk of NK/T cell lymphoma.

\begin{tabular}{|c|c|c|c|c|c|c|c|c|c|c|}
\hline \multirow[b]{2}{*}{ Variable } & \multicolumn{2}{|c|}{$\begin{array}{l}\text { Cases } \\
(\mathrm{N}=417)\end{array}$} & \multicolumn{2}{|c|}{$\begin{array}{l}\text { Controls } \\
(\mathrm{N}=488)\end{array}$} & \multicolumn{3}{|l|}{ Univariable } & \multicolumn{3}{|c|}{ Multivariable } \\
\hline & No. & $\%$ & No. & $\%$ & OR & $95 \% \mathrm{CI}$ & $p$ & $\mathrm{AOR}^{*}$ & $95 \% \mathrm{CI}$ & $p$ \\
\hline anti-HBc-(-) and never drinking & 206 & 49.4 & 289 & 59.2 & & 1(reference) & & & 1(reference) & \\
\hline $\begin{array}{l}\text { anti-HBc-(-) and } \\
\text { ever drinking }\end{array}$ & 37 & 8.9 & 31 & 6.4 & 1.674 & $1.006-2.787$ & 0.047 & 2.013 & $1.124-3.604$ & 0.019 \\
\hline anti-HBc-(+) and never drinking & 146 & 35.0 & 144 & 29.5 & 1.422 & $1.063-1.904$ & 0.018 & 1.598 & $1.149-2.222$ & 0.005 \\
\hline $\begin{array}{l}\text { anti-HBc-(+) and } \\
\text { ever drinking }\end{array}$ & 28 & 6.7 & 24 & 4.9 & 1.637 & $0.922-2.905$ & 0.092 & 2.123 & $1.121-4.020$ & 0.021 \\
\hline
\end{tabular}

The $\mathrm{AOR}^{*}$ was adjusted by sex, age (as a continuous variable), ABO blood type, HBV infection status, smoking status, alcohol drinking and a family history of cancers. Abbreviations: OR, odds ratio; AOR, adjusted odds ratio; 95\% CI, 95\% confidence interval. 
The results of previous case-control studies and meta-analyses have generally supported the presence of an association between HBV and the risk of NHL, especially of the large B cell lymphoma subtype.[26, $27,32]$ Similarly, our data showed that anti-HBc status was associated with ENKTL status, implying that past or previous exposure to HBV was a risk factor for ENKTL diagnosis. However, the potential mechanisms underlying the association between HBV infection and NHL risk are unknown. In a cohort study conducted in Taiwan, Fwu and colleagues reported that the association between chronic HBV infection status and intrahepatic cholangiocarcinoma (ICC) was stronger than the association between chronic HBV infection status and NHL. The authors postulated that the mechanism of HBV pathogenesis may be closely associated with active HBV replication in ICC patients but chronic immune stimulation in NHL patients.[33] Consistent with this hypothesis, our data showed that the odds of ENKTL diagnosis were neither associated with $\mathrm{HBsAg}$ nor $\mathrm{HBeAg}$ seropositivity, both of which serve as markers of viral replication. Therefore we postulated the increase risk of ENKTL was partly due to the indirect role of chronic inflammation induced by HBV. Previous research has shown that HBV infection often induced systemic inflammation, which has been reported to be characterized by increases in the levels of several cytokines.[34] Under inflammatory conditions, proinflammatory mediators are known to stimulate the proliferation of cells and increase the production of reactive oxygen and nitrogen species, thereby resulting in DNA damage and oncogene mutations, such as JAK3 mutation. [9, 35, 36]

Interestingly, in the analysis in which never exposure to HBV was used as the reference group, our results showed that individuals with previous HBV infection were at increased odds of ENKTL diagnosis; however, a similar association was not identified in chronically HBV infected subjects. Previous infection indicates past exposure to and subsequent clearance of HBV, while chronic HBV carriers are persistently HBsAg-seropositive. [37, 38] This result indicates that factors that facilitate HBV clearance may be associated with increased odds of ENKTL development. Recently, Li et al. reported that the rs $9277378^{*} \mathrm{~A}$ risk, which is located in HLA-DPB1, was associated with increased risk of NKTCL, especially among patients who did not have concurrent HBV infection. Previous research has also indicated that HLA-DPB1 SNPs were associated with some inflammatory and immune-mediated diseases and that the rs9277378 SNP was in near-complete linkage disequilibrium with the rs9277535 SNP, which has been found to be associated with HBV clearance. Therefore, the authors suspected that persons with higher HBV clearance ability would be at higher risk of NKTCL development.[39] In our study, we confirmed this hypothesis, finding that subjects with high HBV clearance ability were at greater odds of being diagnosed with ENKTL. Thus, we speculate that the SNPs associated with host immunity and inflammation response to $\mathrm{HBV}$ may play an important role in the development of ENKTL.

The host immune response also plays an important role in HBV clearance. While anti-HBs status was not statistically associated with the odds of ENKTL diagnosis in our study, individuals with natural immunity to $\mathrm{HBV}$ were at increased odds of being diagnosed with ENKTL (AOR, 1.871; 95\% 1.302-2.689). However, similar results were not identified among individuals who lacked an immune response to HBV. This finding is inconsistent with previous data regarding the effect of immune responses on B-cell NHL. Marcucci et al. demonstrated that a lack of immune response was positively associated with B-cell NHL.[25] Kleinstern et al. concluded that patients who lacked an immune response were at increased risk of DLBCL.[32] We propose that immunity may play different roles in the induction and promotion of pathogenesis in different lymphomas because of the discrepancies in their associated genetic mutations, including SNPs.

Ye and colleagues reported that anti-HBc seropositivity might be associated with an increased risk of EBV-related nasopharyngeal development among HBV patients. [30] Previous studies have indicated that ENKTL was strongly associated with EBV.[40, 41] Therefore, it is possible that HBV interacts with EBV to induce ENKTL pathogenesis. However, mechanisms potentially underlying the interaction between HBV and EBV are unknown. It is evident that both of HBV and EBV can efficiently infect B lymphoblastic cells.[20, 42, 43] Though Kurth $\mathrm{J}$ et al. showed that adults carry 1 to $50 \mathrm{EBV}$-infected B cells per $10^{6} \mathrm{~B}$ cells, the EBV enter a state of latency to escape the NK or $\mathrm{T}$ cell-mediated immune response.[44] B-cells can be activated when an individual is infected with $\mathrm{HBV}$,[45] which may potentially activating latent EBV. Furthermore, activated B-cells that are infected with EBV may shed increased numbers of EBV virions,[30] thereby increasing the probability of infection to NK or T cells, which are attempting to kill an EBV-infected cell target.[46]

The study's s strengths include the inclusion of the large cohort of ENKTL patients to date and the examination of several hepatitis biomarkers, enabling differentiation between the effects of HBV exposure, viral clearance and host immune responses on ENKTL 
status. However, there are some limitations in the present research that should be considered. First, we failed to present socio-economic status (SES) information of the patients which is known to be related to the prevalence of $\mathrm{HBV}$ infection in some countries.[47] However, such an effect should be minimal, since previous studies from our area suggest no strong association between the virus prevalence and SES.[48] Second, due to the rarity of ENKTL, we were unable to conduct a population-based prospective trial to evaluate the role of HBV. Third, because HBV-DNA load data were not available for most patients, we could not precisely measure HBV replication.

In conclusion, we conducted a case-control study to identify the association between HBV and ENKTL in an epidemic area. We identified an association between prior HBV infection and ENKTL status. This finding suggests that $\mathrm{HBV}$ clearance and host immune response to HBV may be associated with ENKTL development. Future prospective studies are warranted to confirm these findings, and experimental, epidemiological studies are necessary to reveal the mechanisms underlying this association.

\section{Acknowledgements}

We are grateful to all staffs at Sun Yat-sen University Cancer Center for helping with file management. This work was supported in part by the National Natural Science Foundation of China (Grant number: 81600154) to Kefeng Wang, National Natural Science Foundation of China (Grant number: 81502573), Natural Science Foundation of Guangdong Province (Grant number: 2014A030310421) and Young Teacher Fund of Sun Yat-sen University (Grant number: 16ykpy20) to Panpan Liu.

\section{Competing Interests}

The authors have declared that no competing interest exists.

\section{References}

1. Au WY, Weisenburger DD, Intragumtornchai $T$, Nakamura S, Kim WS, Sng I, et al. Clinical differences between nasal and extranasal natural killer/T-cell lymphoma: a study of 136 cases from the International Peripheral T-Cell Lymphoma Project. Blood. 2009; 113: 3931-7.

2. Tse E, Kwong YL. How I treat NK/T-cell lymphomas. Blood. 2013; 121: 4997-5005.

3. Adams SV, Newcomb PA, Shustov AR. Racial Patterns of Peripheral T-Cell Lymphoma Incidence and Survival in the United States. Journal of clinical oncology : official journal of the American Society of Clinical Oncology. 2016; 34: 963-71.

4. Gaal K, Sun NC, Hernandez AM, Arber DA. Sinonasal NK/T-cell lymphomas in the United States. The American journal of surgical pathology. 2000; 24: 1511-7.

5. Au WY. Current management of nasal NK/T-cell lymphoma. Oncology. 2010; 24: $352-8$.

6. Au WY, Gascoyne RD, Klasa RD, Connors JM, Gallagher RP, Le ND, et al. Incidence and spectrum of non-Hodgkin lymphoma in Chinese migrants to British Columbia. British journal of haematology. 2005; 128: 792-6.
7. Huang Y, de Reynies A, de Leval L, Ghazi B, Martin-Garcia N, Travert M, et al. Gene expression profiling identifies emerging oncogenic pathways operating in extranodal NK/T-cell lymphoma, nasal type. Blood. 2010; 115: 1226-37.

8. Iqbal J, Kucuk C, Deleeuw RJ, Srivastava G, Tam W, Geng H, et al. Genomic analyses reveal global functional alterations that promote tumor growth and novel tumor suppressor genes in natural killer-cell malignancies. Leukemia. 2009; 23: 1139-51.

9. Koo GC, Tan SY, Tang T, Poon SL, Allen GE, Tan L, et al. Janus kinase 3 -activating mutations identified in natural killer/T-cell lymphoma. Cancer discovery. 2012; 2: 591-7.

10. Quintanilla-Martinez L, Kremer M, Keller G, Nathrath M, Gamboa-Dominguez A, Meneses A, et al. p53 Mutations in nasal natural killer/T-cell lymphoma from Mexico: association with large cell morphology and advanced disease. The American journal of pathology. 2001; 159: 2095-105.

11. Ye Z, Cao Q, Niu G, Liang Y, Liu Y, Jiang L, et al. p63 and p53 expression in extranodal NK/T cell lymphoma, nasal type. Journal of clinical pathology. 2013; 66: 676-80.

12. Jiang L, Gu ZH, Yan ZX, Zhao X, Xie YY, Zhang ZG, et al. Exome sequencing identifies somatic mutations of DDX3X in natural killer/T-cell lymphoma. Nature genetics. 2015; 47: 1061-6.

13. Kanno H, Kojya S, Li T, Ohsawa M, Nakatsuka S, Miyaguchi M, et al. Low frequency of HLA-A*0201 allele in patients with Epstein-Barr virus-positive nasal lymphomas with polymorphic reticulosis morphology. International journal of cancer. 2000; 87: 195-9.

14. Kojya S, Matsumura J, Ting L, Hongyo T, Inazawa J, Kirihata M, et al. Familial nasal NK/T-cell lymphoma and pesticide use. American journal of hematology. 2001; 66: 145-7.

15. Xu JX, Hoshida Y, Yang WI, Inohara H, Kubo T, Kim GE, et al. Life-style and environmental factors in the development of nasal NK/T-cell lymphoma: a case-control study in East Asia. International journal of cancer. 2007; 120: 406-10.

16. Kanavaros P, Lescs MC, Briere J, Divine M, Galateau F, Joab I, et al. Nasal T-cell lymphoma: a clinicopathologic entity associated with peculiar phenotype and with Epstein-Barr virus. Blood. 1993; 81: 2688-95.

17. Harabuchi $Y$, Yamanaka N, Kataura A, Imai S, Kinoshita T, Mizuno F, et al. Epstein-Barr virus in nasal T-cell lymphomas in patients with lethal midline granuloma. Lancet. 1990; 335: 128-30.

18. Suzuki R, Yamaguchi M, Izutsu K, Yamamoto G, Takada K, Harabuchi Y, et al. Prospective measurement of Epstein-Barr virus-DNA in plasma and peripheral blood mononuclear cells of extranodal NK/T-cell lymphoma, nasal type. Blood. 2011; 118: 6018-22.

19. Kwong YL, Pang AW, Leung AY, Chim CS, Tse E. Quantification of circulating Epstein-Barr virus DNA in NK/T-cell lymphoma treated with the SMILE protocol: diagnostic and prognostic significance. Leukemia. 2014; 28 : 865-70.

20. Yoffe B, Noonan CA, Melnick JL, Hollinger FB. Hepatitis B virus DNA in mononuclear cells and analysis of cell subsets for the presence of replicative intermediates of viral DNA. The Journal of infectious diseases. 1986; 153: $471-7$.

21. Sun Z, Ming L, Zhu X, Lu J. Prevention and control of hepatitis B in China. Journal of medical virology. 2002; 67: 447-50.

22. Liang X, Bi S, Yang W, Wang L, Cui G, Cui F, et al. Reprint of: Epidemiological serosurvey of Hepatitis B in China--declining HBV prevalence due to Hepatitis B vaccination. Vaccine. 2013; 31 Suppl 9: J21-8.

23. Donato F, Boffetta P, Puoti M. A meta-analysis of epidemiological studies on the combined effect of hepatitis B and $C$ virus infections in causing hepatocellular carcinoma. International journal of cancer. 1998; 75: 347-54.

24. Lee $\mathrm{CH}$, Chang $\mathrm{CJ}$, Lin $\mathrm{YJ}$, Yeh $\mathrm{CN}$, Chen MF, Hsieh SY. Viral hepatitis-associated intrahepatic cholangiocarcinoma shares common disease processes with hepatocellular carcinoma. British journal of cancer. 2009; 100: 1765-70.

25. Marcucci F, Mele A, Spada E, Candido A, Bianco E, Pulsoni A, et al. High prevalence of hepatitis B virus infection in B-cell non-Hodgkin's lymphoma. Haematologica. 2006; 91: 554-7.

26. Engels EA, Cho ER, Jee SH. Hepatitis B virus infection and risk of non-Hodgkin lymphoma in South Korea: a cohort study. The Lancet Oncology. 2010; 11: 827-34.

27. Nath A, Agarwal R, Malhotra P, Varma S. Prevalence of hepatitis B virus infection in non-Hodgkin lymphoma: a systematic review and meta-analysis. Internal medicine journal. 2010; 40: 633-41.

28. Wang L, Wu-Xiao ZJ, Chen XQ, Zhang YJ, Lu Y, Xia ZJ. Hepatitis B virus infection correlates with poor prognosis of extranodal natural killer/T cell lymphoma. Leukemia \& lymphoma. 2015; 56: 936-41.

29. Wu F, Qu L, Tan Y, Zhang Y, Hu C. L-asparaginase-induced severe acute pancreatitis in an adult with extranodal natural killer/T-cell lymphoma, nasal type: A case report and review of the literature. Oncology letters. 2014; 7: 1305-7.

30. Ye YF, Xiang YQ, Fang F, Gao R, Zhang LF, Xie SH, et al. Hepatitis B virus infection and risk of nasopharyngeal carcinoma in southern China. Cancer epidemiology, biomarkers \& prevention : a publication of the American Association for Cancer Research, cosponsored by the American Society of Preventive Oncology. 2015; 24: 1766-73.

31. Hassan MM, Li D, El-Deeb AS, Wolff RA, Bondy ML, Davila M, et al. Association between hepatitis B virus and pancreatic cancer. Journal of clinical 
oncology : official journal of the American Society of Clinical Oncology. 2008; 26: 4557-62.

32. Kleinstern G, Seir RA, Perlman R, Abdeen Z, Khatib A, Elyan H, et al. Associations between B-cell non-Hodgkin lymphoma and exposure, persistence and immune response to hepatitis B. Haematologica. 2016; 101: e303-5.

33. Fwu CW, Chien YC, You SL, Nelson KE, Kirk GD, Kuo HS, et al. Hepatitis B virus infection and risk of intrahepatic cholangiocarcinoma and non-Hodgkin lymphoma: a cohort study of parous women in Taiwan. Hepatology. 2011; 53: 1217-25.

34. Bishayee A. The role of inflammation and liver cancer. Advances in experimental medicine and biology. 2014; 816: 401-35.

35. Sarvaiya PJ, Guo D, Ulasov I, Gabikian P, Lesniak MS. Chemokines in tumor progression and metastasis. Oncotarget. 2013; 4: 2171-85.

36. Ohnishi S, Ma N, Thanan R, Pinlaor S, Hammam O, Murata M, et al. DNA damage in inflammation-related carcinogenesis and cancer stem cells. Oxidative medicine and cellular longevity. 2013; 2013: 387014.

37. Lok AS, McMahon BJ. Chronic hepatitis B. Hepatology. 2007; 45: 507-39.

38. Iloeje UH, Yang HI, Chen CJ. Natural history of chronic hepatitis B: what exactly has REVEAL revealed? Liver international : official journal of the International Association for the Study of the Liver. 2012; 32: 1333-41.

39. Li Z, Xia Y, Feng LN, Chen JR, Li HM, Cui J, et al. Genetic risk of extranodal natural killer T-cell lymphoma: a genome-wide association study. The Lancet Oncology. 2016; 17: 1240-7.

40. Kanakry JA, Hegde AM, Durand CM, Massie AB, Greer AE, Ambinder RF, et al. The clinical significance of EBV DNA in the plasma and peripheral blood mononuclear cells of patients with or without EBV diseases. Blood. 2016; 127: 2007-17.

41. Gru AA, Haverkos BH, Freud AG, Hastings J, Nowacki NB, Barrionuevo C, et al. The Epstein-Barr Virus (EBV) in T Cell and NK Cell Lymphomas: Time for a Reassessment. Current hematologic malignancy reports. 2015; 10: 456-67.

42. Li Q, Spriggs MK, Kovats S, Turk SM, Comeau MR, Nepom B, et al. Epstein-Barr virus uses HLA class II as a cofactor for infection of B lymphocytes. Journal of virology. 1997; 71: 4657-62.

43. Babcock GJ, Decker LL, Volk M, Thorley-Lawson DA. EBV persistence in memory B cells in vivo. Immunity. 1998; 9: 395-404

44. Kurth J, Spieker T, Wustrow J, Strickler GJ, Hansmann LM, Rajewsky K, et al. EBV-infected B cells in infectious mononucleosis: viral strategies for spreading in the B cell compartment and establishing latency. Immunity. 2000; 13: 485-95.

45. Suarez F, Lortholary O, Hermine O, Lecuit M. Infection-associated lymphomas derived from marginal zone B cells: a model of antigen-driven lymphoproliferation. Blood. 2006; 107: 3034-44.

46. Tabiasco J, Vercellone A, Meggetto F, Hudrisier D, Brousset P, Fournie JJ. Acquisition of viral receptor by NK cells through immunological synapse. Journal of immunology. 2003; 170: 5993-8.

47. Akbar N, Basuki B, Mulyanto, Garabrant DH, Sulaiman A, Noer HM. Ethnicity, socioeconomic status, transfusions and risk of hepatitis B and hepatitis C infection. Journal of gastroenterology and hepatology. 1997; 12: 752-7.

48. Zeng F, Guo P, Huang Y, Xin W, Du Z, Zhu S, et al. Epidemiology of hepatitis $B$ virus infection: results from a community-based study of 0.15 million residents in South China. Scientific reports. 2016; 6: 36186. 